\title{
Monitoring and Instantaneous Evaluation of Composite Resin Linear Shrinkage with Computerized Topography
}

Amer A Sultan

BDS, MSc (Asst. Lec.)

\section{Department of Conservative Dentistry}

College of Dentistry, University of Mosul

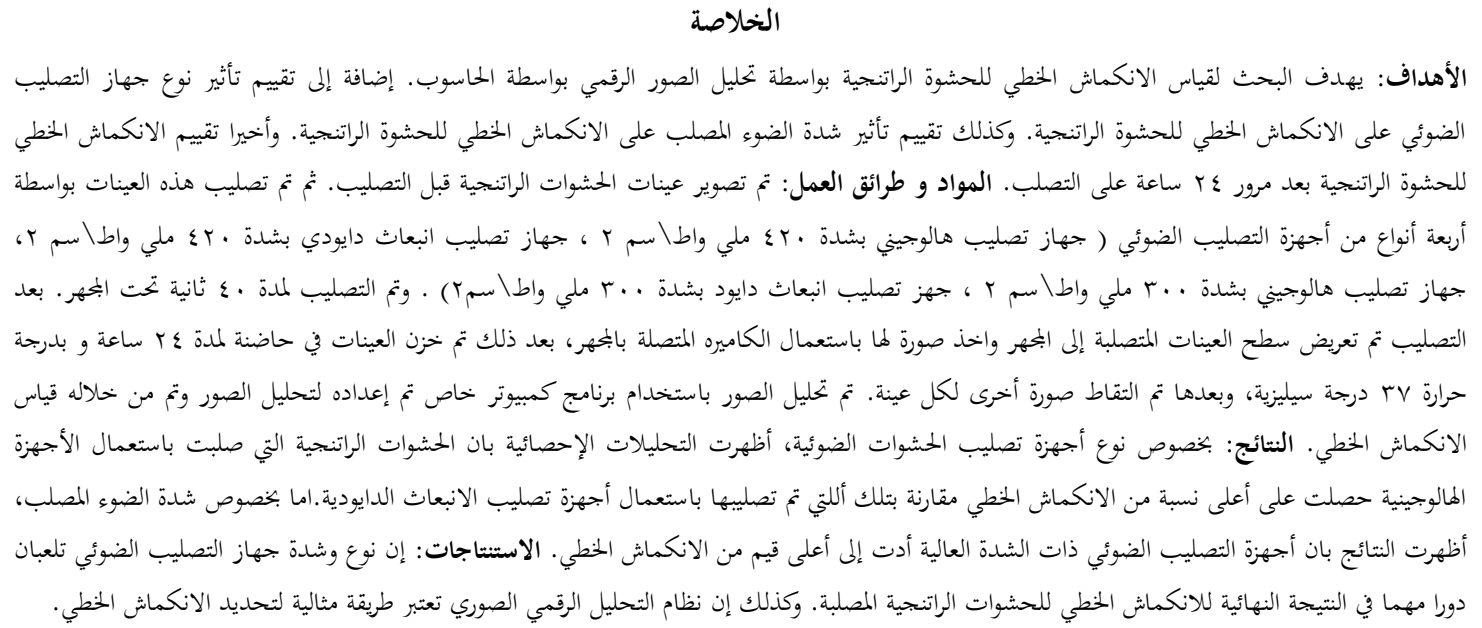

\section{ABSTRACT}

Aims: To measure the linear shrinkage of composite resin by digital image analysis, to evaluate the effect of light curing type on composite resin linear shrinkage, to evaluate the effect of light curing intensity on linear shrinkage of composite resin and to evaluate the linear shrinkage of composite resin after 24 hours. Materials and method: images of composite samples were obtained before curing. The samples were cured with four types of light curing units, halogen light curing unit $420 \mathrm{~mW} / \mathrm{cm} 2$, LED light curing unit $420 \mathrm{~mW} / \mathrm{cm} 2$, halogen light curing $300 \mathrm{~mW} / \mathrm{cm} 2$ and LED light curing unit 300 $\mathrm{mW} / \mathrm{cm} 2$. the curing time was 40 seconds. After curing, the cured composite surface was exposed to the microscope allowing the attached camera to capture an image. The samples were then stored in 37 degree centigrade incubator for 24 hours and another image for each sample, was obtained. The images were analyzed via specially prepared image analysis program and the linear shrinkage was measured. Results: Regarding the type of LCUs, statistical analysis showed that composite samples cured with QTH LCUs exhibit significantly higher linear shrinkage values than those cured with LED LCUs. While regarding the intensity of the curing light, it was shown that LCUs with higher curing light intensities produce a significant higher values of linear shrinkage. Conclusion: The type and the intensity of the light curing unit play a major role in the end result linear shrinkage of the cured composite resin. Image analysis system is an excellent method to determine linear shrinkage.

Keywords: Composite resin, photopolymerization, linear shrinkage.

Sultan AA. Monitoring and Instantaneous Evaluation of Composite Resin Linear Shrinkage with Computerized Topography. Al-Rafidain Dent J. 2013; 13(1): 102-107.

Received: $22 / 5 / 2011 \quad$ Sent to Referees: $30 / 5 / 2011$

Accepted for Publication: 10/10/2011

\section{INTRODUCTION}

Dental composites are increasingly being used for dental restorations because of their good workability and esthetic appearance. However, the biggest disadvantage of resin composites is that polymer-based materials shrink during polymerization. ${ }^{(1-3)}$

During photopolymerization of dime thacrylate-based composites, the transformation of an initially viscous material into a viscoelastic solid is accompanied by a significant volumetric shrinkage and increase in elastic modulus. ${ }^{(4-6)}$

The polymerization shrinkage of composite materials has been implicated as a primary source of interfacial breakdown, resulting in visible white lines or invisible 
cracks in the enamel and resin at the margins. ${ }^{(7)}$ It also has been blamed for the tooth-composite interfacial debonding, cuspal deflection, enamel cracking, and the formation of white lines. ${ }^{(8)}$ The bond failure at the interface allows an influx of oral fluids and greatly contributes to the possibility of marginal staining, secondary caries, postoperative sensitivity, and fractures through the enamel along the interface. ${ }^{(9)}$

There exist different methods of measuring the shrinkage of materials. One method is by determining the density change of the material by Archimedes's method, using buoyancy and weight in different liquids. The pyknometer is based on this method. ${ }^{(10)}$

One system that actually measures the volume change is the mercury dilatometer, which records the changes in volume by observing height changes in a capillary column. ${ }^{(11)}$

The bonded disc method is another method which can measure the volumetric shrinkage of dental composites to a good approximation. With this method, a thin disc of the dental composite is bonded to a rigid lower glass plate while a thin piece of glass diaphragm is bonded to the top surface of the composite disc. The deflection of the diaphragm caused by the shrinking composite during polymerization is then measured using a linear voltage differential transducer (LVDT). ${ }^{(12)}$

A newer system that measures volume changes is based on optical measurements and pattern recognition. This systems including image collection, finding averages images of the specimen, does edge detection on the image, and then calculates the volume of the specimen. The change in volume is reported as a percentage shrinkage. The system is a non contact system, as opposed to the mercury dilatometer, avoiding the hazard of air inclusions ${ }^{(13,14)}$.

The aims of this study where to measure the linear shrinkage of composite resin by digital image analysis, to evaluate the effect of light curing type on composite resin linear shrinkage, to evaluate the ef- fect of light curing intensity on linear shrinkage of composite resin and to evaluate the linear shrinkage of composite resin after 24 hours.

\section{MATERIALS AND METHODS}

Stainless steel metal molds of $(4 \times 4) \mathrm{mm}$ (in length and width) and $2 \mathrm{~mm}$ in depth were used to prepare 40 composite samples, the type of the composite resin used in this study was (SAREMCO, Saremco Dental AG, Brazil ).

The samples were divided into four groups, the first group was cured with halogen light curing unit (Astralis, Vivadent, Austria) with the intensity of 420 $\mathrm{mW} / \mathrm{cm}^{2}$.

The second group was cured with LED light curing unit (ULTRA-LITE 200E PLUS, TAIWAN) with the intensity of $420 \mathrm{~mW} / \mathrm{cm}^{2}$. the third group was cured with CUTE-LIGHT 1E (TAIWAN) with the intensity of $300 \mathrm{~mW} / \mathrm{cm}^{2}$.

The fourth group was cured with the same halogen light curing unit, but at the intensity of $300 \mathrm{~mW} / \mathrm{cm}^{2}$. The intensity of the halogen light curing unit was adjusted via a custom made resistant volume switch in order to match the intensity of the LED light curing unit.

For the purpose of holding the metal molds used in this study, a special rectangular metal base was fabricated. Each of the metal molds were fixed at this metal base by the use of elastic threads at a time, the light curing unit also was fixed at this metal base in such a way that the curing tip is at right angle to the side of the metal mold with $\mathrm{a}(1 \mathrm{~cm})$ distance between the curing tip and the side surface of the composite resin samples( the surface subjected to the curing light). The metal base was then placed under the lens of the stereo microscope (Heerbrugg, Switzerland), in such a way that the lens is perpendicular to the top surface of the composite samples (the surface which will be subjected to the measurement of linear shrinkage) Figure(1). 


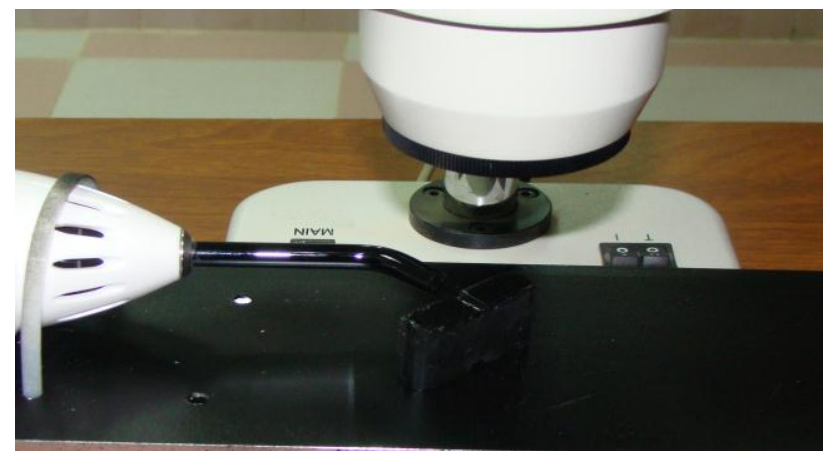

Figure (1): Metal base (black) holding the composite mold and the light cure unit under the stereo microscope.

The distance between light curing unit and the side surface of the composite sample, and the distance between the lens of the stereo microscope and the top surface of the composite sample, were measured and controlled by digital caliper (CHINA).

Before curing of the composite samples, an image was captured for each sample and labeled as a pre cured sample.

Curing of the samples were done under the stereo microscope at one side surface of the sample and for 40 seconds, during which, the top surface of the composite sample was covered by a black glass slap. After curing the cover was immediately removed, exposing the cured composite surface to the microscope allowing the attached camera to capture an image (the resolution of the attached camera was $(1024 \times 768)$, these images were labeled as immediate post cured samples.

The samples were then stored in 37 degree centigrade incubator (Memmert Gmbh +COKG, Germany) for 24 hours in dry condition, after which the samples were brought to the stereo microscope and another image for each sample, was obtained, and labeled as 24 hours post cured samples.
The images were analyzed via specially prepared image analysis software via (ImageJ software version $1.44 \mathrm{p}$ Wayne Rasband) ${ }^{(15)}$ that include a primary enhancement process of the image, and a series of programmed steps that were designed to calculate the difference between the pre and post cured composite samples per pixels.

The accuracy of the prepared program was verified through the use of a cured composite sample $4 \times 4 \times 2$. An image of this sample was obtained after curing, followed by a reduction of $1 \mathrm{~mm}$ from one end of the sample, the reduction was performed via diamond disc attached to a milling machine (1000 MAX MILLING MACHINE, BRAZIL). Then another image of the sample after reduction was obtained.

Both of the pre and post reduction images, were subjected to the image analysis program, and the results confirmed an excellent accuracy level $(99.8 \%)$.

Finally the linear shrinkage was measured from the difference between the pre and post cured composite sample images as shown in Figure (2), and its values were expressed in percentages.

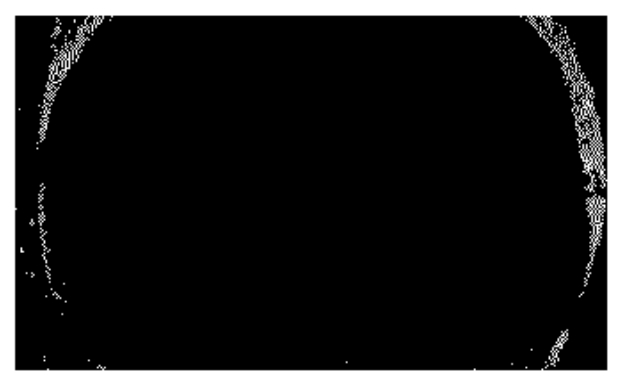

Figure (2): The post cured image super imposed the pre cured image where white dotes represent the amount of linear shrinkage. 


\section{RESULTS}

Statistical analysis was conducted on the data obtained from image analysis program. The mean linear polymerization shrinkage values for samples cured with different types and different intensities light curing units, immediately after curing and 24 hours after curing, are shown in Table (1), Figure (2).

Table (1): Mean and Standard deviationof linear shrinkage

\begin{tabular}{llll}
\hline \multicolumn{1}{c}{ Composite samples } & Mean of linear shrinkage & $\mathrm{N}$ & Std. Deviation \\
\hline Post Cure High Halogen & 2.8540 & 10 & .08343 \\
Post Cure High LED & 2.2920 & 10 & .11708 \\
Post Cure Low Halogen & 2.4682 & 10 & .01631 \\
Post Cure Low LED & 2.0869 & 10 & .01664 \\
24H Post Cure High Halogen & .1840 & 10 & .00699 \\
24H Post Cure High LED & .1820 & 10 & .00789 \\
24H Post Cure Low Halogen & .1823 & 10 & .00340 \\
24H Post Cure Low LED & .1800 & 10 & .00000 \\
& 1.3037 & 80 & 1.14742 \\
\hline
\end{tabular}

In addition, results showed that there is a significant difference in the values of immediate linear polymerization shrinkage between the composite samples cured with halogen light at both high and low intensities, and those cured with LED light curing units (high and low intensities) Table (2).

Table (2): Duncan's test results for linear shrinkage

\begin{tabular}{lllllll}
\hline Variables & \multicolumn{7}{c}{ Subset for alpha = 0.05 } & \\
& $\mathrm{N}$ & 1 & 2 & 3 & 4 & 5 \\
\hline 24 H Post cure Low LED & 10 & .1800 & & & & \\
24 H Post cure Low Halogen & 10 & .1820 & & & & \\
24 H Post cure High LED & 10 & .1823 & & & & \\
24 H Post cure High Halogen & 10 & .1840 & & & & \\
Post cure Low LED & 10 & & 2.0869 & & & \\
Post cure High LED & 10 & & & 2.2920 & & \\
Post cure Low Halogen & 10 & & & & 2.4682 & \\
Post cure High Halogen & 10 & & & & & 2.8540 \\
& & .877 & 1.000 & 1.000 & 1.000 & 1.000 \\
\hline
\end{tabular}

\section{DISCUSSION}

The polymerization of dental resins is invariably accompanied by the volumetric shrinkage of the cured material compared with the unset state. Shrinkage is associated with the polymerization reaction in a complex way. ${ }^{(16)}$

When monomers in proximity react to establish a covalent bond, the distance between the two groups of atoms is reduced and there is a reduction in free volume, both of which translate into volumetric shrinkage. ${ }^{(17)}$

In the pre-gel phase, the polymer chains formed are very flexible so that material of the free surface of the cavity can flow. The viscosity of the developing polymer is still low, so that shrinkage stress can be compensated for. The time at which the material is no longer able to compensate for polymerization contraction (time until gelation or vitification) therefore, determines the final stress in the material. ${ }^{(18)}$

Plastic deformation or flow of the composite resin may occur to a limited extent, until vitrification occurs. Reduced light intensity may result in a storage modulus development at a slow enough rate to allow for flow and dissipation of stress. As the setting process proceeds, shrinkage and flow decrease gradually because storage modulus increases. ${ }^{(19,20)}$ 
Under high light intensities, composite resins restrain this stress relief much more by not allowing enough flow to reduce internal stress. Restriction of the flow capacity by the configuration of the restoration also enhances the contraction stress leading in return to increase volumetric shrinkage. ${ }^{(18,19)}$

Asmussen et al. explains that a slow polymerization start with low intensity is associated with relatively few centers of polymer growth resulting in a more linear polymer structure with relatively few cross-links. On the other hand, a high intensity during the initial phase of the irradiation period will initiate a multitude of growth centers and a polymer with higher cross-link density results. ${ }^{(21,22)}$

These beneficial effects of flow and decreased numbers of polymer growth, are being utilized when decreased light intensity is employed and explain why the LCUs with low intensities produce a significant decrease in linear shrinkage. ${ }^{(18)}$

For direct filling materials, the temperature differential generated during photopolymerization is one of the most important factors that affect the polymerization shrinkage. ${ }^{(23)}$

The vinyl addition polymerization reaction is exothermic by approximately $55 \mathrm{~kJ} / \mathrm{mol}$, based on the methacrylate functional group. Compounding this effect, the heat generated from the tungsten halogen light curing units, will greatly increase the polymerization shrinkage by increasing the mobility of free radicals within the polymeric network and allows free monomer and pendant chains to encounter remaining radical sites and react further. And hence the explanation for the increase of shrinkage values with QTH LCUs regardless of the curing intensities. . $^{23)}$

Another consequence of radicals trapped within polymers is the protracted post-cure, or additional conversion, that continues after irradiation is ended. The majority of potential conversion that can be attained with a given resin composition is achieved during the first few minutes after irradiation; however, an increase in both conversion and, particularly, the evolution of polymer properties are observed up to and beyond 24 hours. ${ }^{(23)}$
The non significant difference in the values of 24 hours post-cure, linear shrinkage of composite resin samples cured with different types of LCUs at different intensities can be explained by the kinetic theory of photopolymerization. All composite samples will reach the vitrificatioin point (point at which the material transitioned from rubbery to glassy polymer) at the end of curing cycle regardless of the type or intensity of the curing light. Studies have shown that, even with the present of free trapped radicals that tend to further react and produce the protracted post-cure, the remaining unreacted monomer exhibit decrease reactivity beyond the vitrification point, becoming more mobile and more hydrophilic and rapidly leaches from dental composite. ${ }^{(23,24)}$

\section{CONCLUSION}

The type and the intensity of the light curing unit play a major role in the end result linear shrinkage of the cured composite resin. Light curing units that promote heat generation (QTH) lead to an increase in the total amount of composite shrinkage. In addition, the higher intensity of the LCU, result in the higher shrinkage values. Image analysis system is an excellent method to determine linear shrinkage in a non contact procedure that do not affect the other properties of the cured composite samples.

\section{REFRENCES}

1. Watts DC, Kisumbi BK, Toworfe GK. Dimensional changes of resin/ionomer restoratives in aqueous and neutral media. Dent Mater. 2000;16: 89-96.

2. Ensaff H, O'Doherty DM, Jacobsen PH. Polymerization shrinkage of dental composite resins. Proc Inst Mech Eng [H] 2001; 215:367-75.

3. Watts DC, Marouf AS, Al-Hindi AM. Photo-polymerization shrinkage-stress kinetics in resin-composites: methods development. Dent Mater. 2003; 19:111.

4. Calheiros FC, Braga RR, Kawano Y, Ballester RY. Relationship between contraction stress and degree of conversion in restorative composites. Dent Mater. 2004; 20(10):939-46. 
5. Emami N, Soderholm KJ, Berglund LA. Effect of light power density variations on bulk curing properties of dental composites. J Dent. 2003; 31(3): 189-96.

6. Halvorson RH, Erickson RL, Davidson CL. Energy dependent polymerization of resin-based composite. Dent Mater. 2002; 18(6): 463-9.

7. Ferrracane JL. Placing. Dental composites-a stressful experience. Oper Dent. 2008; 33-34: 247-257.

8. Van Noort R. Introduction to Dental Materials. London: Mosby; 2002.

9. O'Brien WJ. Dental Materials and Their Selection. Chicago, IL: Quintessence Publishing Co, Inc; 2002.

10.Sargent Welch Co. Selective experiments in physics. http://www.sargentwelch.com/info. Accessed November 26, 2008.

11.Gee AJ, Davidson CL. A modified dilatometer for continuous recording of volumetric polymerization shrinkage of composite restorative materials. J Dent. 1981; 9: 36-42.

12. Watts DC, Cash AJ. Determination of polymerization shrinkage kinetics in visible-light-cured materials: methods development. Dental Materials 1991;7:281-7.

13. Sharp LJ, Choi IB, Lee TE, et al. Volumetric shrinkage of composites using video imaging. J Dent. 2003; 31(2): 97 103.

14.Tiba A, Charlton D, Vandewalle K, Ragain J Jr. Comparison of two videoimaging instruments for measuring volumetric shrinkage of dental resin components. J Dent. 2005; 33(9):757763.

15.ImageJ software version $1.44 p$ Wayne Rasband, National Institute of Health,
USA, http://imageJ. Nih, gov/ij.

16. Feilzer A, de Gee A. Influence of light intensity on polymerization shrinkage and integrity of restoration-cavity interface. Eur J Oral Sci. 1995;103:322-6.

17.Watts DC, Cash A. Determination of polymerization shrinkage kinetics in visible light-cured materials: materials development. Dent Mater. 1991;7: 2817.

18. Roberto R. Bragaa,, Rafael Y. Ballestera, Jack L. Ferracaneb Factors involved in the development of polymerization shrinkage stress inresincomposites: A systematic review Dent Mater. (2005); 21: 962-970

19.Uno S, Asmussen E. Marginal adaptation of a restorative resin polymerized at reduced rate. Scand J Dent Res. 1991; 99:440-4.

20.Uno S, Asmussen E. Marginal adaptation of a restorative resin polymerized at reduced rate. Scand J Dent Res. 1991; 99: 440-4.

21.Asmussen E, Peutzfeldt A. Influence of pulse-delay curing on softening of polymer structures. J Dent Res. 2001; 80: 1570-3.

22.Asmussen E, Peutzfeldt A. Two-step curing: influence on conversion and softening of a dental polymer. Dent Mater. 2003;19: 466-70.

23.Jeffrey W.stansbury; curing dental resins and composites by photopolymerization. J Esthetic Dent. 2000; 12: 300308 ,

24.A Jeffery W. Stansbury, Marianela Trujillo-lemon, Hui Lu, Xingzhe Ding, Yan Lin, Junhao Ge. Conversiondependat shrinkage stress and strain in dental resins and composites. Dent Mater. (2005); 21: 56-67. 